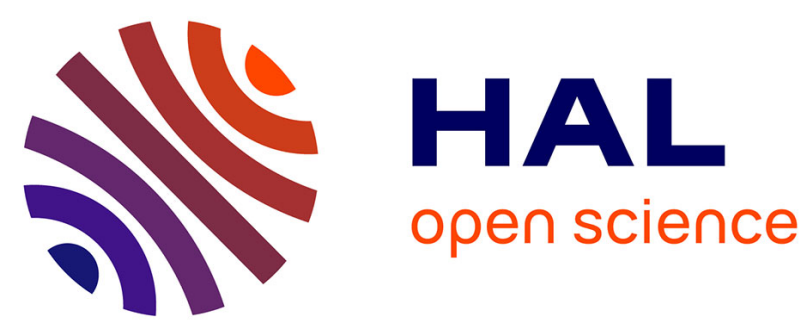

\title{
Hydration Phase Diagram of Clay Particles from Molecular Simulations
}

Túlio Honório, Laurent Brochard, Matthieu Vandamme

\section{To cite this version:}

Túlio Honório, Laurent Brochard, Matthieu Vandamme. Hydration Phase Diagram of Clay Particles from Molecular Simulations. Langmuir, 2017, 33 (44), pp.12766-12776. 10.1021/acs.langmuir.7b03198 . hal-01695174

\section{HAL Id: hal-01695174 https://hal.science/hal-01695174}

Submitted on 20 Jun 2018

HAL is a multi-disciplinary open access archive for the deposit and dissemination of scientific research documents, whether they are published or not. The documents may come from teaching and research institutions in France or abroad, or from public or private research centers.
L'archive ouverte pluridisciplinaire HAL, est destinée au dépôt et à la diffusion de documents scientifiques de niveau recherche, publiés ou non, émanant des établissements d'enseignement et de recherche français ou étrangers, des laboratoires publics ou privés. 


\title{
Hydration phase diagram of clay particles from molecular simulations
}

\author{
Tulio Honorio,* Laurent Brochard, and Matthieu Vandamme \\ Université Paris-Est, Laboratoire Navier (UMR 8205), CNRS, ENPC, IFSTTAR, $6 \& 8$ \\ Avenue Blaise Pascal, 77455 Marne-la-Vallée, France \\ E-mail: tulio.honorio-de-faria@enpc.fr;tuliohfaria@gmail.com
}

Phone: +(33) 164153659

\begin{abstract}
Adsorption plays a fundamental role in the behavior of clays. Due to the confinement between clay solid layers at the nanoscale, adsorbed water is structured in layers, which can occupy a specific volume. The transition between these states is intimately related to key features of clay thermo-hydro-mechanical behavior. In this paper, we consider the hydration states of clays as phases and the transition between these states, as phase changes. The thermodynamic formulation supporting this idea is presented. Then, results from grand canonical Monte Carlo simulations of sodium-montmorillonite are used to derive hydration phase diagrams. The stability analysis presented here explains the coexistence of different hydration states at clay particle scale and improves our understanding of the irreversibilities of clay thermo-hydro-mechanical behavior. Our results provide insights into the mechanics of the elementary constituents of clays, which is crucial for a better understanding of the macroscopic behavior of clay-rich rocks and soils.
\end{abstract}




\section{Introduction}

Clay is one of the most abundant class of materials in the Earth's crust and plays an important role in a variety of industrial applications (notably, energy production) and environmental processes. ${ }^{1}$ Smectites are a type of clay that is present in abundance in terrestrial and marine environments. ${ }^{1}$ The structure of clay minerals is composed of negatively charged layers of colloidal size. These layers are composed of octahedral sheets interposed between two tetrahedral sheets. Due to substitutions in both sheets, the layers may exhibit a net charge. In the interlayer space, cations of different natures are present in order to compensate layers' charge. Water is allowed to ingress in the interlayer space due to the moderate charge of layers $\left(-0.1\right.$ to $\left.-0.2 \mathrm{C} / \mathrm{m}^{2}\right) .{ }^{1}$ The swelling of the structure results from the water uptake. Due to the confinement in the interlayer space, adsorbed water is structured in layers corresponding to specific basal spacings $d$ (i.e. the center-to-center distance between solid layers). Dehydrated $(d=9.7-10.2 \AA)$, mono-hydrated $(d=11.6-12.9 \AA)$, bi-hydrated $(d=14.9-15.7 \AA)$ and tri-hydrated $(d=18-19 \AA)$ states, often referred to as $0 \mathrm{~W}, 1 \mathrm{~W}, 2 \mathrm{~W}$ and $3 \mathrm{~W}$, respectively, can be identified for different types of clays ${ }^{1-3}$. For large pore sizes, a pore water (or capillary water) hydration state $(\infty \mathrm{W})$ can also be defined. Note, however, that large pore sizes may also be due to interparticle pores. ${ }^{4,5}$

The hydration of smectites depends on the nature (e.g. size and charge) of the counterion, temperature, confining pressure and pressure of the fluid reservoir. The identification of the hydration states according to these conditions is crucial with respect to the determination of clay thermo-hydro-mechanical behavior. Since each hydration state is associated with a volume and water content, the volume changes in clayed materials are intimately related

to transitions in hydration states. Recently, the authors ${ }^{6,7}$ have shown that changes in hydration states can explain the thermal expansion/contraction of clay (clay exhibits thermal expansion or contraction that are reversible or irreversible depending on their loading history). A key element in this analysis was the central role of hysteresis in clay swelling, as recognized previously by different authors. For example, Verburg et al. ${ }^{8}$ studied the hystere- 
sis accompanying cation exchanges and pointed out the role of activation energy barriers in the swelling at the layer scale. Boek et al. ${ }^{9}$ discussed the way in which the irreversibilities in clay swelling are intimately related to hysteresis. Other authors ${ }^{10-14}$ treated hydration states as thermodynamic phases and the volume changes in the material as resulting from phase transitions.

In this spirit, hydration phase diagrams were proposed by $\operatorname{Bird}^{10}$ taking into account confining pressures, pressures of water reservoir and temperatures of interest for oil extraction. This author employed generalized Clapeyron law associated with an experimental tests in order to get the phase diagrams. The results are, therefore, based in continuum thermodynamics, which might not capture some specificities of adsorption and interface effects within clays. Ferrage et al. ${ }^{15}$ proposed hydration phase diagrams of Na-smectite based on X-ray diffraction and adsorption/desorption experiments at ambient temperature according to different relative humidities. Emmerich et al. ${ }^{16}$ presented hydration phase diagrams for Na-montmorillonites with two different structural charges. Their results investigate different relative humidities under ambient confining pressure. Other studies are focused rather on compositional aspects ${ }^{17}$ than on the variables of interest in geomechanics.

A practical application of hydration phase diagrams of clay is to determine the conditions for which clay remains stable. These diagrams may also contribute to a better understanding of the propensity of clays to swelling for conditions of interest in industrial applications and in environmental processes. In this sense, multiscale investigations of the thermo-mechanical behavior of clay may use as input the information from hydration phase diagrams. Additionally, with the use of molecular simulations, the necessary information to build the hydration phase diagrams can be obtained under controlled conditions regarding mixed interlayer counterions and mixed hydration states. Also, these simulations allow to explicitly account for water structuration within interlayers pores leading to an accurate description of hydration phase diagrams.

The goal of this study is to construct the hydration phase diagram of a saturated swelling 
clay (sodium montmorillonite, or Na-Mmt) based on molecular simulations. By "saturated" clay, we mean that the chemical potential and/or pressure of water remain superior to the ones associated with (liquid) saturation. Grand-Canonical Monte Carlo (GCMC) simulations are performed to obtain Na-Mmt confining pressure isotherms according to a range of temperatures and pressures of water reservoir of interest in geomechanical applications. We identify stable basal spacings and the domains of metastability through the analysis of confining pressure isotherms and corresponding free energy of swelling. To do so, the appropriate thermodynamic potentials are defined. The theoretical framework to cope with phase transitions for materials experiencing volume changes is presented. Our results explain the coexistence of different hydration states at clay particle scale and provide kinetics arguments, based on the energy barriers to be overcome in transformations, leading to the hysteresis observed in response to mechanical loading. Finally, the stability analysis allows drawing a phase diagram of the (meta-)stable hydration states of clay particles as a function of temperature and the confining pressure of bulk water.

\section{Materials and methods}

\section{Molecular simulation methods}

GCMC simulations of sodium-saturated montmorillonite are performed in order to mimic drained conditions, in which clay interlayer pores are in equilibrium with an external water reservoir. We choose montmorillonite due to its propensity to swell. Additionally, montmorillonite is a well-studied system in molecular simulations; ${ }^{18-24}$ this provides basis for benchmarking some aspects of our simulations.

Based on previous works, ${ }^{25,26}$ the adopted atomic structure of montmorillonite is built from the structure of pyrophyllite determined by X-ray diffraction. ${ }^{27}$ Montmorillonite is obtained by substitutions, in a layer, of one out of each eight octahedral aluminium atoms by a magnesium atom and one out of each 32 tetrahedral silicon atoms by an aluminium 
atom. The substitutions obey Loewenstein rule, ${ }^{28}$ i.e. substitution sites are not adjacent to each other. The simulation super-cell is obtained by replicating the layers in each direction of the unit cell. The resulting triclinic super-cell has two slit pores. For sodium montmorillonite, the resulting structural formula is $\mathrm{Na}_{6}^{+} \cdot\left[\mathrm{Si}_{62} \mathrm{Al}_{2}\right]\left[\mathrm{Mg}_{4} \mathrm{Al}_{28}\right] \mathrm{O}_{160}(\mathrm{OH})_{32} \cdot \mathrm{nH}_{2} \mathrm{O}$.

The non-bonded interactions between atoms are described by Lennard-Jones and Coulomb potentials:

$$
U\left(r_{i j}\right)=4 \epsilon_{i j}\left[\left(\frac{\sigma_{i j}}{r_{i j}}\right)^{12}-\left(\frac{\sigma_{i j}}{r_{i j}}\right)^{6}\right]+\frac{q_{i} q_{j}}{4 \pi \epsilon_{0} r_{i j}}
$$

where $r_{i j}$ is the distance between the center of atoms $i$ and $j ; \epsilon_{i j}$ and $\sigma_{i j}$ are Lennard-jonnes parameters regarding the interaction between atoms $i$ and $j, q_{i}$ is the partial charge of a given atom $i$ and $\epsilon_{0}$ is the permittivity. Lennard-Jonnes parameters and partial charges are obtained from the ClayFF force field ${ }^{29}$ together with the extended simple point charge (SPC/E) model of water. ${ }^{30}$ Lennard-Jones cross terms are derived following Lorentz-Berthelot mixing rules. Simulations are run with periodic boundary conditions. A cutoff distance of $8.9 \AA$ is adopted for short range dispersive interactions. Ewald's summation method (with accuracy in forces of $10^{-4}$ ) is employed for long range electrostatic interactions.

The total surface charge density of the layers is -0.124 C.m², which is compensated by the appropriate number of cations in the interlayer space in order to ensure electroneutrality.

The structure was relaxed with LAMMPS package ${ }^{31}$ considering the harmonic bonds and angles parameters defined in ClayFF for water and hydroxyls. After relaxation at $300 \mathrm{~K}$ and $1 \mathrm{~atm}$, the simulation super-cell has the in-plane dimensions $L_{a}=20.72 \AA$ and $L_{b}=17.96$ A. Since the thermal expansion and compressibility of fluids (even in a confined state) are expected to be much higher than that of solids, the effect of the deformation of the solid lattice due to the thermal expansion or compression should be minor. Regarding size effects, larger supercells with a more disordered distribution of the isomorphic substitutions would be necessary to contour size dependence of the thermodynamic and structural output of simulations. Note however that in the case of cesium-montmorillonite, the specific locations 
of isomorphic substitutions have shown only a minor effect on thermodynamic, structural (including the basal spacing) and transport properties of the system for a fixed clay composition and layer charge. ${ }^{19}$ Small differences in hydration energy of mono-hydrated states was observed, though; and the differences may be accentuated in the case of higher layer charge and higher counterion valence. ${ }^{19}$

For GCMC simulations, we assume that the effects related to the flexibility of both the solid lattice and water molecules are negligible. Therefore, clay layers and water molecules are kept rigid in order to gain in computational efficiency. Thus, only non-bonded interactions of ClayFF are needed. Simulations in grand canonical ensemble were performed with Towhee ${ }^{32}$ package using configurational-bias methods. Biased insertion/deletion (with a probability $p=0.37$ for water molecules), rotation (for water molecules $p=0.09$ with a maximum angle $=\pi$ rad) and translation ( $p=0.37$ for both ion and water molecule, with maximum displacement $=$ $1.5 \AA$ ) Monte Carlo moves as well as swaps ( $p=0.11$ for water molecules and ions) and growth ( $p=0.06$ for water molecules) moves are employed. During the simulation, the interlayer space is filled with water according to different basal spacings in response to a given chemical potential of water. This chemical potential is chosen in order to reflect a target water pressure consistent with some environmental conditions of interest in geomechanics. Only the number of water molecules is allowed to fluctuate in the simulation box as the sodium counterions are treated in the canonical ensemble, i.e. each ion was allowed to move, while the number of ions remained constant. For each temperature and basal spacing, equilibration is performed over $10^{7} \mathrm{MC}$ steps and sampling during $2 \times 10^{7} \mathrm{MC}$ steps. Our convergence criterion is a stable energy system (with the standard deviation of the total energy below $0.02 \%$ of the mean value).

The confining pressure is computed by means of the virial formula as discussed in ${ }^{33}$ (chapter 2), which is the default method of computing pressure in most MC simulations. ${ }^{32}$ In our GCMC simulations, the entire super-cell is considered in the computation of the pressure, even if the interactions inside the solid are not taken into account since the solid is 
kept rigid. In this sense, adjustments of the computed pressure with respect to the pore size may be proposed in the form $P_{a d j}=P / \phi$. For a volume $V=A$.d, the porosity is $\phi=h_{p} / d$, where $h_{p}=d-h_{s}$ is the thickness of the pore and $h_{s}$, the thickness of the solid layer. The latter can be defined as the distance $d_{o}$ between the out-most atoms, on the layer surface, projected to $z$-axis. Other possibility is to defined $h_{s}$ as $d_{o}$ plus the van der Waals radius of each of the out-most atoms. ${ }^{34}$ We prefer not to introduce such adjustments provided that the choice of the pore size would be arbitrary.

A prerequisite of our study is the determination of the chemical potential of water $\mu_{w}$ corresponding to a given temperature $T$ and water pressure $P_{w}$ of the reservoir. Simulations of bulk water in grand canonical ensemble can be used to determine $\mu_{w}$, but high pressure fluctuations are expected. For instance, Smith et al. ${ }^{35}$ reported variations in the target pressure of circa $200 \mathrm{MPa}$ for a fixed $\mu_{w}$ with SPC/E water. Our strategy was to combine simulations of bulk SPC/E water in canonical and grand canonical ensembles in order to obtain $\mu_{w}$ as a function of the water pressure $P_{w}$ and temperature T. Molecular dynamics simulations in canonical ensemble are performed with LAMMPS ${ }^{31}$ using SHAKE algorithm to rigidify water molecules. Systems with 300 water molecules were equilibrated during 1 ns and sampled during 2 ns. For a given temperature, $\mu_{w}$ is obtained by thermodynamic integration using Gibbs-Duhem equation: $\left.\frac{\partial \mu_{w}}{\partial P_{w}}\right|_{T}=\frac{1}{\rho_{w}}$, with $\rho_{w}$ being the density of water. The integration is performed starting from the saturation point of SPC/E water (see gray dashed lines in Figure 1) for which the chemical potential is known accurately through Transition Matrix Monte Carlo (TMMC) simulations. ${ }^{36}$ We performed also TMMC simulations of bulk $\mathrm{SPC} / \mathrm{E}$ water with Towhee. For these, the system was equilibrated during $10^{7} \mathrm{MC}$ moves and sampling during $2 \times 10^{7} \mathrm{MC}$ biased moves (as described in the last paragraph). Figure 1 shows the chemical potential of SPC/E water for different temperatures. The outputs of $\mu \mathrm{VT}$ and NVT simulations are in a reasonable agreement. At $300 \mathrm{~K}$ and $1 \mathrm{~atm}$, the chemical potential of bulk SPC/E water is reported ${ }^{36,37}$ to be $\mu_{w}=-49.0 \mathrm{~kJ} / \mathrm{mol}$, which is in accord with our estimations. 

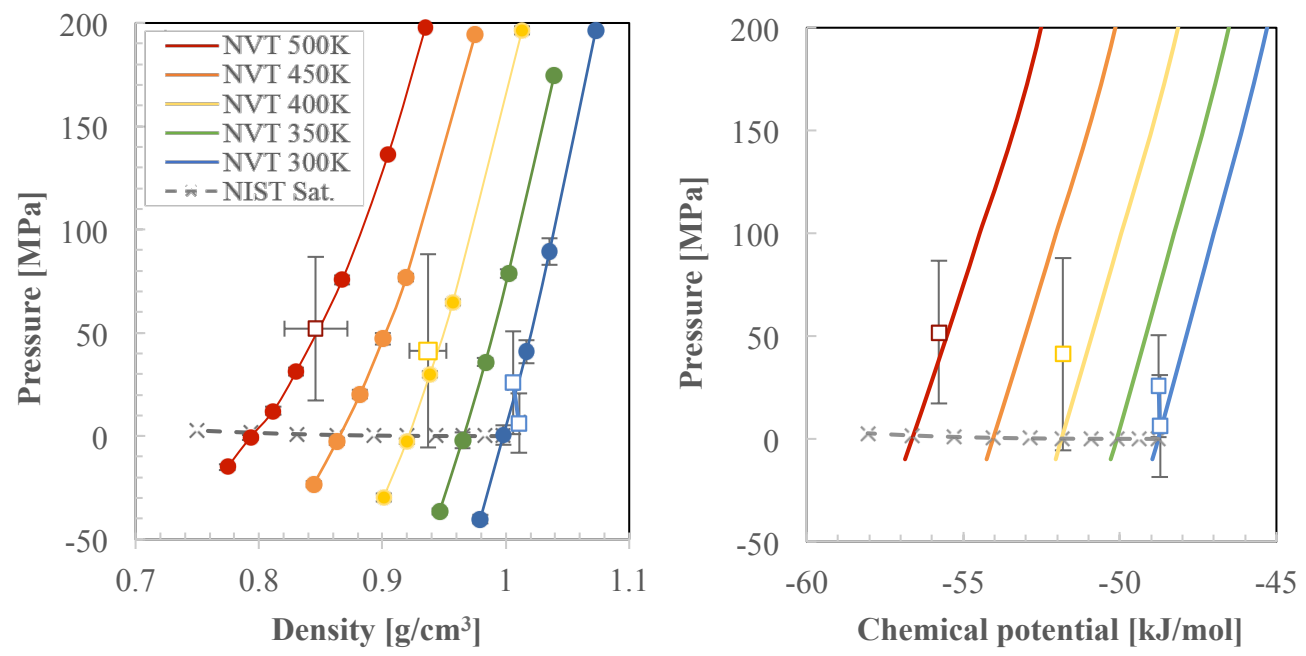

Figure 1: Pressure isotherms (left) and chemical potential (right) of bulk SPC/E water for different temperatures according to thermodynamic integration of pressure isotherms obtained from canonical simulation of bulk water (solid lines). We carried out thermodynamic integration from saturation points of SPC/E water ${ }^{36}$ (shown in the pictures with the gray crosses). The squares correspond to TMMC simulations. 


\section{Thermodynamics of swelling and stability of clay layers and particles}

In this section, we consider the thermodynamic potentials mimicking drained conditions in clay layers, where adsorption occurs. Then, we analyze the scale of a stack of layers (or clay particle), which is the first structural unit in clayed material generally considered after the clay layers. The examination of this scale can provide some important insight into the hydration states transitions. ${ }^{6}$

\section{Thermodynamic potentials at clay layers scale}

Solid layers, composed of the tetrahedral and octahedral sheets, intercalated by water and counterions are the basic structural units of clays. In drained experimental conditions, water in clay interlayer pores is in equilibrium with an external water reservoir. The thermodynamic ensemble mimicking these conditions is the Grand Canonical ensemble (here $\mu_{w} \mathrm{VT}$ ), in which the chemical potential of water, the volume and the temperature are imposed, while the amount of water, the confining stress and the entropy are fluctuating. The number of counterions and of atoms in the solid are kept constant. In this hybrid ensemble, the system minimizes a mixed thermodynamic potential $\Lambda$ composed of the Helmholtz free energy $F$ of the solid skeleton and counterions plus the grand potential of the water:

$$
\Lambda\left(\mu_{w}, V, T\right)=F-\mu_{w} N_{w}
$$

where $N_{w}$ is the number of water molecules.

In layered materials, the volume per unit of surface $A$ is equal to the basal spacing $d$, i.e. $V / A=d$. In this work, we investigate only dimensional changes in basal spacing $d$ while the surface area $A$ is kept constant. Accordingly, we limit our analysis to the study of the potential per unit area: $\lambda\left(\mu_{w}, d, T\right)=\frac{\Lambda}{A}$. The confining pressure $P$ acting in the direction

orthogonal to the basal plane (or the solid layers) is defined as $P=-\left.\frac{1}{A} \frac{\partial \Lambda}{\partial d}\right|_{T, \mu_{w}}$. The energy profile of $\lambda$ with respect to changes in basal spacing can be obtained by the integration of 
the confining pressure:

$$
\lambda\left(d, T, \mu_{w}\right)=\lambda\left(d_{0}, T, \mu_{w}\right)-\int_{d_{0}}^{d} \operatorname{Pd}(d)
$$

where $d_{0}$ is a reference basal spacing.

Instead of controlling the volume, one may be interested in a system controlled by the confining pressure, whose conjugate variable is $d$. The appropriate thermodynamic potential in this case is a Legendre transform of $\lambda$ :

$$
\lambda^{*}=\lambda+P d
$$

$\Lambda^{*}=\lambda^{*} A$ is the thermodynamic potential that the system minimizes at equilibrium under imposed $\mu_{w}, P$ and $T$. This complementary energy is often called swelling free energy in the literature ${ }^{14,23,38}$. Another potential used to study adsorption in thin films is the osmotic potential (e.g. ${ }^{39}$ ) for which the confining pressure $P$ is set equal to water pressure $P_{w}$ (also called unjacketed conditions in poromechanics). Here, we consider the swelling free energy because it is more general by allowing the independent control of $\mu_{w}, P$ and $T$.

In adsorbing layered materials, typical confining pressure isotherms are oscillating curves with decaying amplitudes versus basal spacing (see e.g. Figure 3 for clays or Brochard et al. ${ }^{6}$ for a general layered adsorbing material). In clays, the dehydrated state corresponds to the first branch of the curve in which the confining pressure strongly increases with the decrease of $d$ due to steric repulsion between clay layers as well as the counterions in the interlayer space. The very first oscillation corresponds to the monohydrated state; the subsequent $i$ oscillations correspond to the $i$-hydrated states up to the pore water state. Accordingly, the swelling energies also exhbit a multi-well configuration when plotted versus basal spacing (see e.g. Figure 7). This is a key element in hydration transitions as shown in the next section. 


\section{Themodynamic potentials at clay particle scale}

Materials with a multi-well potential $\lambda$ are prone to develop a heterogeneous microstructure in order to minimize their total energy. ${ }^{40}$ In this section, we show how the coexistence of hydration states and the stability of clays can be understood as the minimization of the mesoscopic energy associated with a clay particle. ${ }^{40,41}$

Clays are multiscale hierarchical materials. This aspect is relevant to our study since the transition of hydration states occurs with respect to a elementary volume comprising some stacks of layers. Experimental evidence (e.g. Ferrage et al. ${ }^{15}$ with X-Ray diffraction results) shows that different hydration states can coexist according to a variety of environmental conditions. This coexistence is associated with a scale of a few tens of nanometers. ${ }^{15}$ With clays, at least one mesoscopic scale is often identified for the purpose of understanding some aspects of macroscopic behavior of the material. ${ }^{42}$ After the single layers, a stack of layers (with orientational correlations) is the first structural unit identified in clayed materials. Different experimental ${ }^{43-48}$ and theoretical ${ }^{49,50}$ investigations, covering a variety of illites, smectites and other phyllosilicates, reported that the average number of solid layers per particle is from few units up to few tens of layers.

Let us consider a Representative Volume Element (RVE) $\Omega$ within a particle. We define the mesoscopic energy $\hat{\lambda}$ of this RVE as the minimization of the microscopic energy $\lambda$ over $\Omega$, with imposed average deformation $\varepsilon$ of the RVE (or equivalently, for layered materials, the average basal spacing $<d>$ ) on the boundary $\partial \Omega$ of the RVE:

$$
\hat{\lambda}\left(<d>, T, \mu_{w}\right) \equiv \inf _{<d>\text { on } \partial \Omega} \int_{\Omega} \lambda\left(d, T, \mu_{w}\right) d x
$$

where a discrete sum over the stack of layers is approximated by an integral over the RVE. If the solution of this minimization problem corresponds to a homogeneous basal spacing $<d>=d$; such basal spacing is stable (i.e. it really exists in the scale of a layer). The energy $\hat{\lambda}$ is the convex hull of $\lambda$ and includes all the possible stable states of $\Omega$ under 
displacement control. In the portions of $\lambda$ not belonging to $\hat{\lambda}$, a deformation (or average basal spacing) $\left\langle d_{C}>\right.$ laying in-between two stable states $d_{A}$ and $d_{B}$ (i.e. the RVE is heterogeneous and contains different zones with basal spacings $d_{A}$ and $d_{B}$ ) is accommodated within the particle by mixing the stable states (see Figure 2). Indeed, we can write $<d_{C}>=$ $f_{A} d_{A}+\left(1-f_{A}\right) d_{B}$ with $f_{A} \in[0,1]$ being the fraction of material in state $d_{A}$. This phenomenon is a proper characteristic of materials presenting a multi-well energy such as Shape-Memory Alloys (SMA). Figure 2 shows a schematic representation of the combination of stable states in response to a given imposed displacement. The minimization of the mesoscopic energy by mixture of stable states is deemed to lead to an infinitely fine microstructure (i.e. the intercalation of different phases pursuits so that the thickness of the zones corresponding to a given phase tends to zero). ${ }^{40} \mathrm{~A}$ minimal characteristic length is, though, present in real materials and specific arguments can be used to define this length. ${ }^{40}$ In clay systems, we may claim that the minimal characteristic length is the basal spacing in dehydrated state. Also, SMA are subjected to particular crystallographic constraints of compatibility at the interfaces between phases. In contrast, under the assumptions of 1D kinematics for perfectly parallel, infinite and rigid clay layers, such constraints are not imposed onto the interfaces between phases in clay layers. Interface phenomena between neighbouring but not stacked layers (e.g. a edge-to-face configuration) may further penalize the energy barrier to be overcome in phase transitions. The same may take place due to bending of clay layers and particles as discussed in details by the authors elsewhere. ${ }^{50}$

The coexistence of hydration states in clays is, therefore, expected in a system under volume control. The experimental results revealing the coexistence of hydration states refer to powders subject to atmospheric pressure only. ${ }^{15}$ We note that even under macroscopic pressure control, the coexistence may be observed as a results of the prestresses in the microscopic scales. These prestresses may be due to the mismatch of mechanical properties of the different constituent phases in composite materials.

The considerations in this section allow defining a stable state equation between confining 


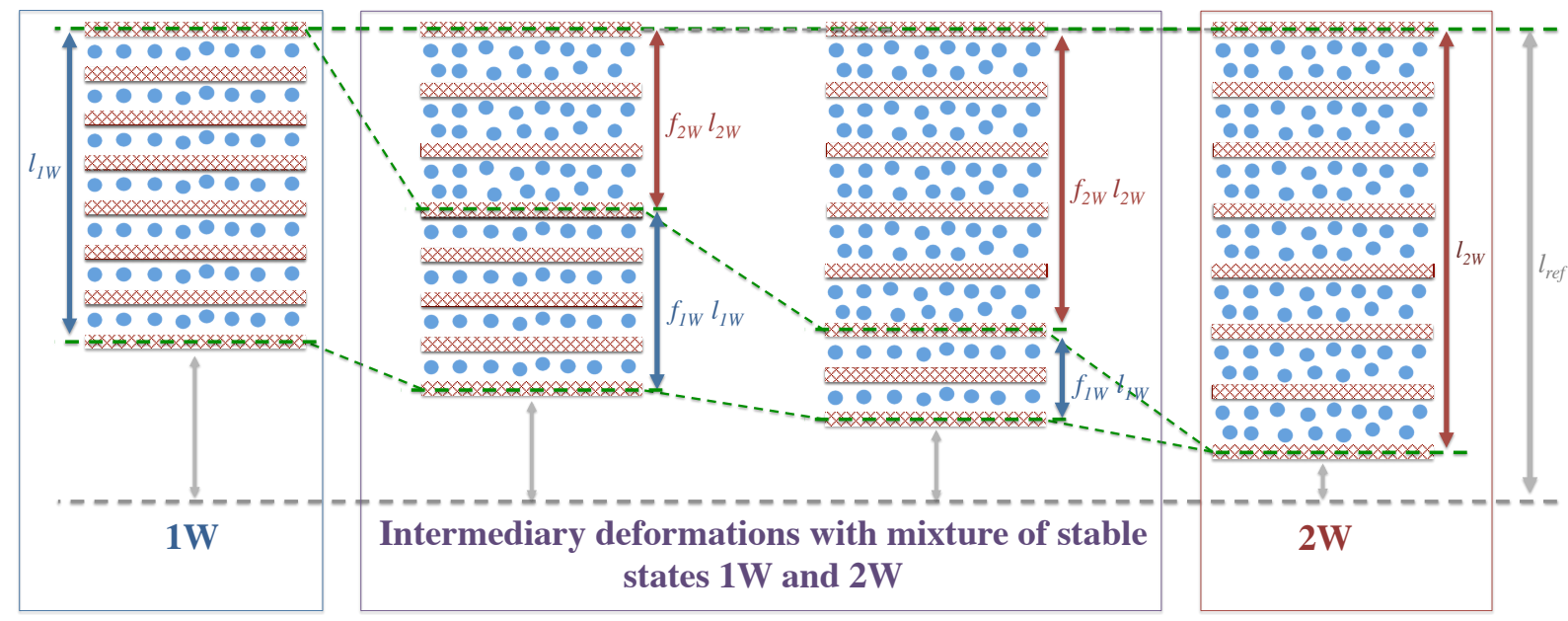

Figure 2: Sketches of stacks with deformation corresponding to stable $1 \mathrm{~W}$ and $2 \mathrm{~W}$ states $\left(l_{1 W}=i . d_{1 W}\right.$ and $l_{2 W}=i . d_{2 W}$, respectively, where $i$ is the number of layers) and arbitrary intermediary deformation with mixture of $1 \mathrm{~W}$ and $2 \mathrm{~W}$ hydration states, with $f_{1 W}$ and $f_{2 W}=$ $1-f_{1 W}$ being the fraction of layers in $1 \mathrm{~W}$ and $2 \mathrm{~W}$ states, respectively. ${ }^{51} \mathrm{~A}$ length $l_{\text {ref }}$ can be defined as a reference to the measure of volume changes.

pressure and basal spacing based on the transitions between stable states. A single path, called hereafter stable path, is defined for expansion and shrinkage, i.e. no hysteresis is captured. Metastable paths, capturing the irreversibilities of clays behaviour, can also be derived as discussed in the next section.

\section{Metastability and energy barriers}

The consideration of metastability at clay layer scale have been introduced in attempts to explain the origin of hysteresis in clay behavior. ${ }^{14}$ The energy barrier to be overcome in phase transformation between two stable states is obtained by means of the swelling free energy $\lambda^{*}$. Tambach et al. ${ }^{14}$ reported energy barriers in hydration state transitions for montmorillonites on the order of a few $k T / \mathrm{nm}^{2}$. Thus, on the timescales of interest in industrial processes involving clays, even for a $1 \mathrm{~nm}^{2}$ particle, the thermal fluctuations of the system might not be enough to carry the system over the energy barriers associated with hydration state transitions. Provided the size of clay layers (in-plane dimensions ranging from 100 to 1000 $\mathrm{nm}),{ }^{34}$ hydration state transitions likely involve bending of the layers in such a way that 
only small portions of the layers exceed the energy barriers. ${ }^{49}$ Theoretical considerations ${ }^{49}$ and experimental evidences ${ }^{52}$ indicate that clay layers bend according to transition regions with a characteristic length on the order of magnitude of a nanometer. Even with bending occurring over such short lengths, the energy barrier seems sufficient for metastability to hold.

The upper limit of metastable zones are defined by the basal spacings $d_{S}$ for which spinodal decomposition occurs: $\left.\frac{\partial^{2} \lambda}{\partial d^{2}}\right|_{d=d_{s}}=\left.\frac{\partial^{2} \lambda^{*}}{\partial d^{2}}\right|_{d=d_{s}}=0$. The energy barriers to be overcome in phase transitions are a function of the control confining pressure. For $P$ equal to the pressure of stable phase transformation $P_{\text {Sta }}$, both stable states have the same $\lambda^{*}$, which are the local minima, and the energy barrier is maximum. With $P$ equal to the pressure at the spinodal points $P_{S p i}^{-}$or $P_{S p i}^{+}$(with the superscripts + and - standing for loading and unloading, respectively), the energy barrier is zero and $\lambda^{*}$ is monotonic.

The energy barriers $\Delta \Lambda^{*}=A \Delta \lambda^{*}$ of a (meta-)stable state provide information on the prevalence of a given hydration state under a given loading as well as kinetic information. For $\Delta \Lambda^{*}$ on the order of magnitude of the thermal fluctuations $(\approx k T)$, even small perturbations in the system can lead to a transition to a state with lower energy. Following transition state theory, ${ }^{53}$ the time necessary to overcome a given free energy barrier $\left(\Delta \Lambda^{*}\right.$, here) scales with $\exp \left[-\Delta \Lambda^{*} /(k T)\right]$. The prevalence of (meta-)stable states can be, thus, compared according to the loading type.

On short time scales, the consideration of metastable states may be important whilst in long time scales (as those associated with geological processes) the behavior can be viewed as following the stable path. 


\section{Results and Discussion}

\section{Confining and disjoining pressure isotherms}

We performed simulations with different water pressure $P_{w}$ at fixed temperatures. Figure 3 displays the disjoining pressure $\Pi=P(d)-P_{w}$ as a function of the basal spacing at 300 and $500 \mathrm{~K}$ for three values of $P_{w}$. Due to the difficulty of determination of $\mu_{w}$ corresponding to a target water pressure $P_{w}, P_{w}$ is estimated from the average of the computed confining pressures of the last $2 \AA$ simulated basal spacings. Indeed, with $d>20 \AA$, it can be assumed that the confining pressure fluctuates around a value corresponding to $P_{w}$.

Our simulations yield results, in terms of water content and density profiles of species in the interlayer pores, which are in agreement with results in the literature (e.g. ${ }^{14}$ ). Figure 4 shows the density profiles of water hydrogens and oxygen ( $\mathrm{Hw}$ and $\mathrm{Ow}$, respectively) and sodium counterions at stable basal spacings corresponding to $0 \mathrm{~W}, 1 \mathrm{~W}$ and $2 \mathrm{~W}$ states, as well as snapshots of configurations corresponding to these states. These results are in agreement with other density histograms of Na-Mmt in the literature using ClayFF ${ }^{21}$ as well as early work with other force fields. ${ }^{14,54,55}$ The structuration of water molecules is evident according to these density profiles. The insets of Figure 3 shows the water content as a function of the basal spacing. In our analysis, only 0W (first branch), 1W (first oscillation) and 2W (second oscillation) states can be clearly identified; larger basal spacings will be considered as pore water $(\infty \mathrm{W})$. Note that $3 \mathrm{~W}$ state is often reported in experimental studies. ${ }^{56}$

Differences in $\Pi$ obtained at any given water pressure $P_{w}$ are not significant with respect to the amplitude of the oscillations associated with $1 \mathrm{~W}$ and $2 \mathrm{~W}$ states. These differences have the same order of magnitude as the fluctuations observed in the molecular simulations. The same is observed for the other temperatures. Along these lines, $\Pi$ depends only on the basal spacing and temperature, and not on the water pressure $P_{w}$, i.e. $\Pi\left(P_{w}, d, T\right)=\Pi(d, T)$,

as observed also by other authors. ${ }^{10}$ Thus, the confining pressure isotherms for different $P_{w}$ can be readily obtained from one single pressure isotherm. In other words, the deformation in 
the system is controlled by the so-called Terzaghi stress (e.g. ${ }^{57}$ ). Therefore, according to our results, the apparent Biot coefficient of the clay layer defined $\operatorname{as}^{58} b=\left.\frac{\partial P}{\partial P_{w}}\right|_{d}$ is approximately 1 for saturated clays (recall that, in this paper, saturated stands for the chemical potential and pressure of water remaining above the ones associated with liquid saturation). On the other hand, note that unsaturated clays may exhibit apparent Biot coefficients larger that 1 (e.g. $\left.{ }^{59}\right)$
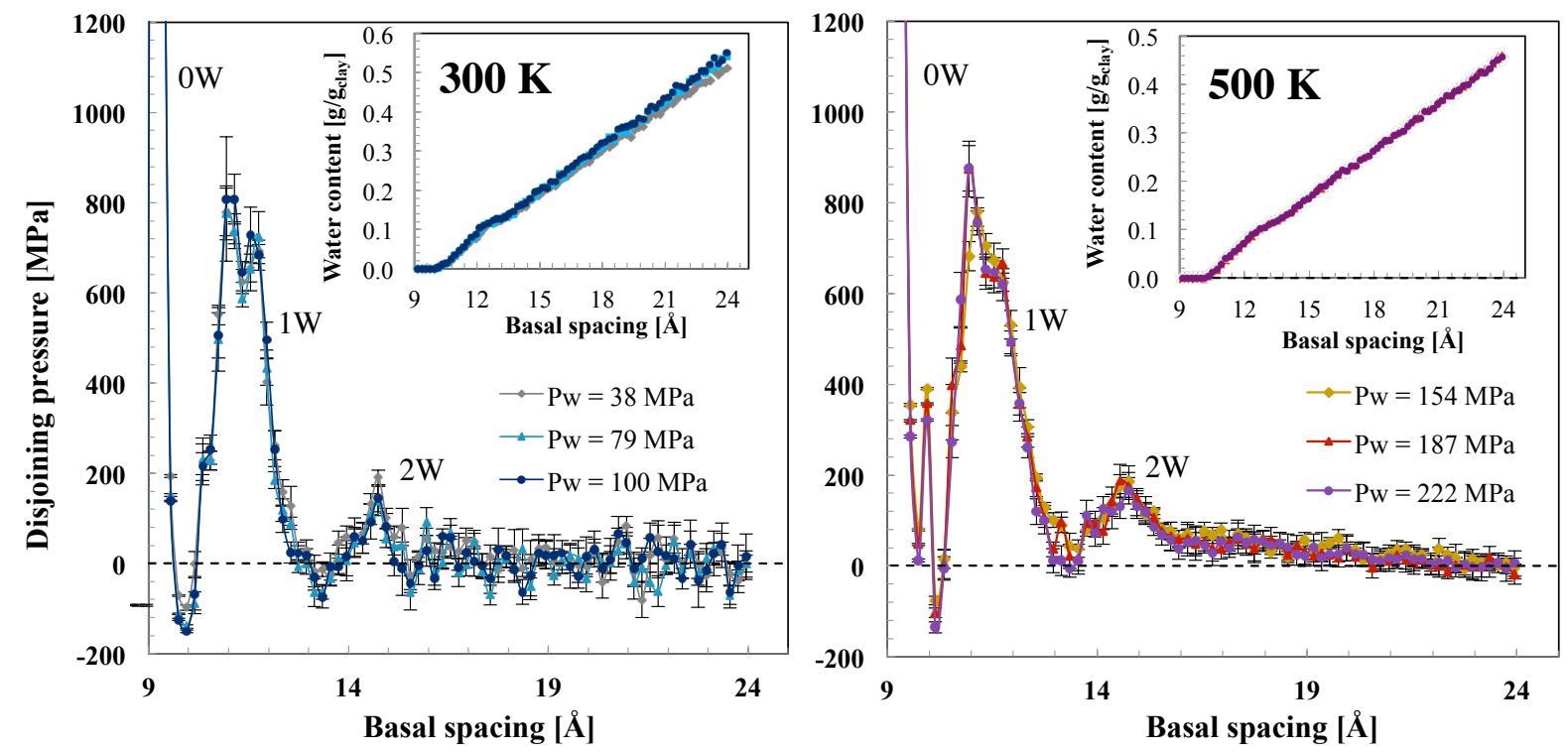

Figure 3: Disjoining pressure isotherms of Na-Mmt at 300 and $500 \mathrm{~K}$ for different water pressures $P_{w}$. The insets display the water content of Na-Mmt at both temperatures for the same values of $P_{w}$.

Figure 5 (top) shows the disjoining pressure isotherms at different temperatures as a function of the basal spacing $d$. The data were smoothed with Savitzky-Golay filter. ${ }^{60}$ The peaks associated with $1 \mathrm{~W}$ (first oscillation) and 2W (second oscillation) states are clearly identified for all the temperatures considered. For all temperatures, the pressure isotherms seem to fluctuate around water pressure $P_{w}$ after $d>20 \AA$. A previous study of the authors ${ }^{6}$ showed that, with Lennard-Jones fluids confined between 9-3 Lennard-Jones walls, the amplitudes of oscillations globaly decrease with the temperature and this effects is more pronounced for larger basal spacings. ${ }^{6}$ Here, the amplitudes of $2 \mathrm{~W}$ states clearly decrease with the temperature, whereas the amplitudes of $1 \mathrm{~W}$ states at different temeprature are quite 

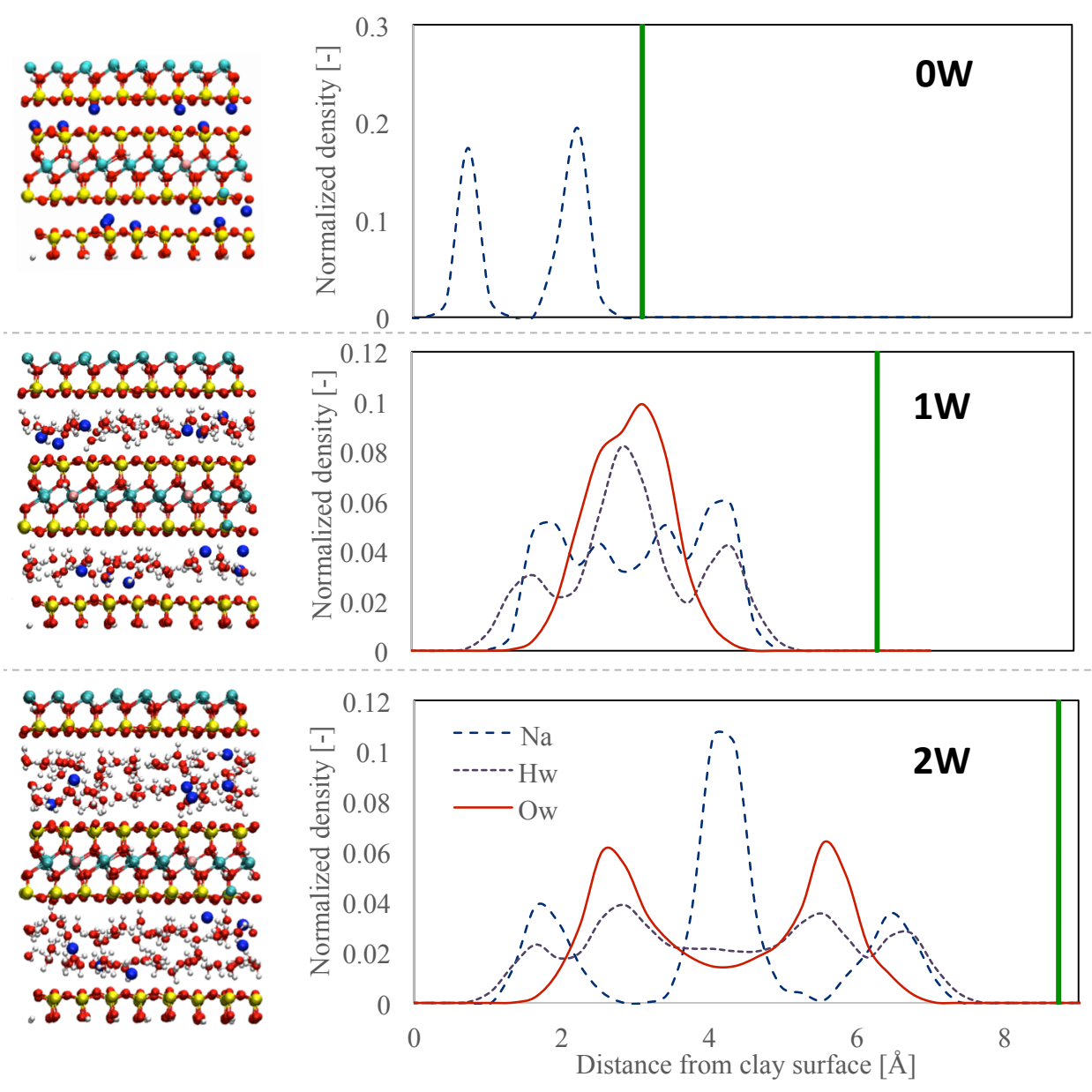

Figure 4: Density histograms and snapshots of configurations showing water and counterion ordering adjacent to clay surface. Density profiles and configurations are taken at stable basal spacings corresponding to $0 \mathrm{~W}, 1 \mathrm{~W}$ and $2 \mathrm{~W}$ states $(d=9.55,12.75$ and $15.15 \AA$, respectively). The density profiles of $\mathrm{Na}$ counterions, water oxygen $(\mathrm{Ow})$ and water hydrogen $(\mathrm{Hw})$ are computed accounting for both slit pores shown at the left. The green lines refer to the position of the opposed clay solid surface. 
similar (as also observed for $1 \mathrm{~W}$ state in LJ fluids ${ }^{6}$ ). In the same work with Lennard-Jones fluids, the peaks of pressure isotherms sligthly glide towards larger basal spacings with the increase of temperature. Here, this effect could not be clearly observed within the precision of our simulations.
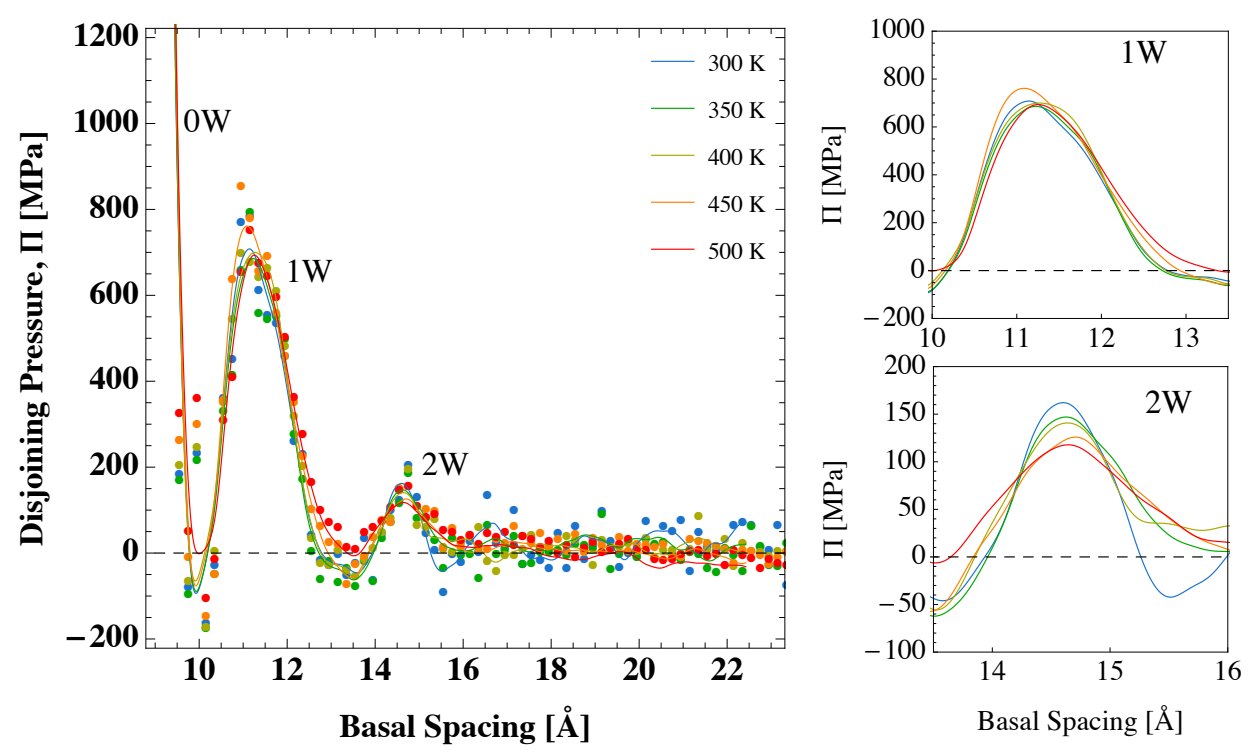

Figure 5: Disjoining pressure isotherms of Na-Mmt at different temperatures as a function of the basal spacing $d$. The solid lines are obtained by Savitzky-Golay smoothing filter. ${ }^{60}$ On the right, the details of the peaks related to $1 \mathrm{~W}$ and $2 \mathrm{~W}$ states.

\section{Free energy of swelling}

Figure 6 (left) displays the microscopic energy $\lambda$ and its convex hull (dashed lines) at 300 $\mathrm{K}$ under different water pressures $P_{w}$; the corresponding stable paths in confining pressure isotherms are also shown. Stable phase transition occurs at constant confining pressure. The confining pressure isotherms are just shifted with respect to water pressure $P_{w}$. The range of basal spacing corresponding to stable states remain approximately the same for all $P_{w}$.

Figure 6 (right) shows the microscopic energy $\lambda$ and its convex hull as well as the isotherm pressures according to temperatures from 300 up to $500 \mathrm{~K}$ under a fluid reservoir pressure 
$P_{w}$ of $10 \mathrm{MPa}$. The pressures $P_{S t a}$ in which phase transition occurs are gathered in Table 1; no clear tendency with respect to the temperature is observed as differences are of the order of the accuracy in pressure. Again, as can be seen in Table 1 comparing the pressures in a given transition, the confining pressure is just shifted with respect to $P_{w}$.
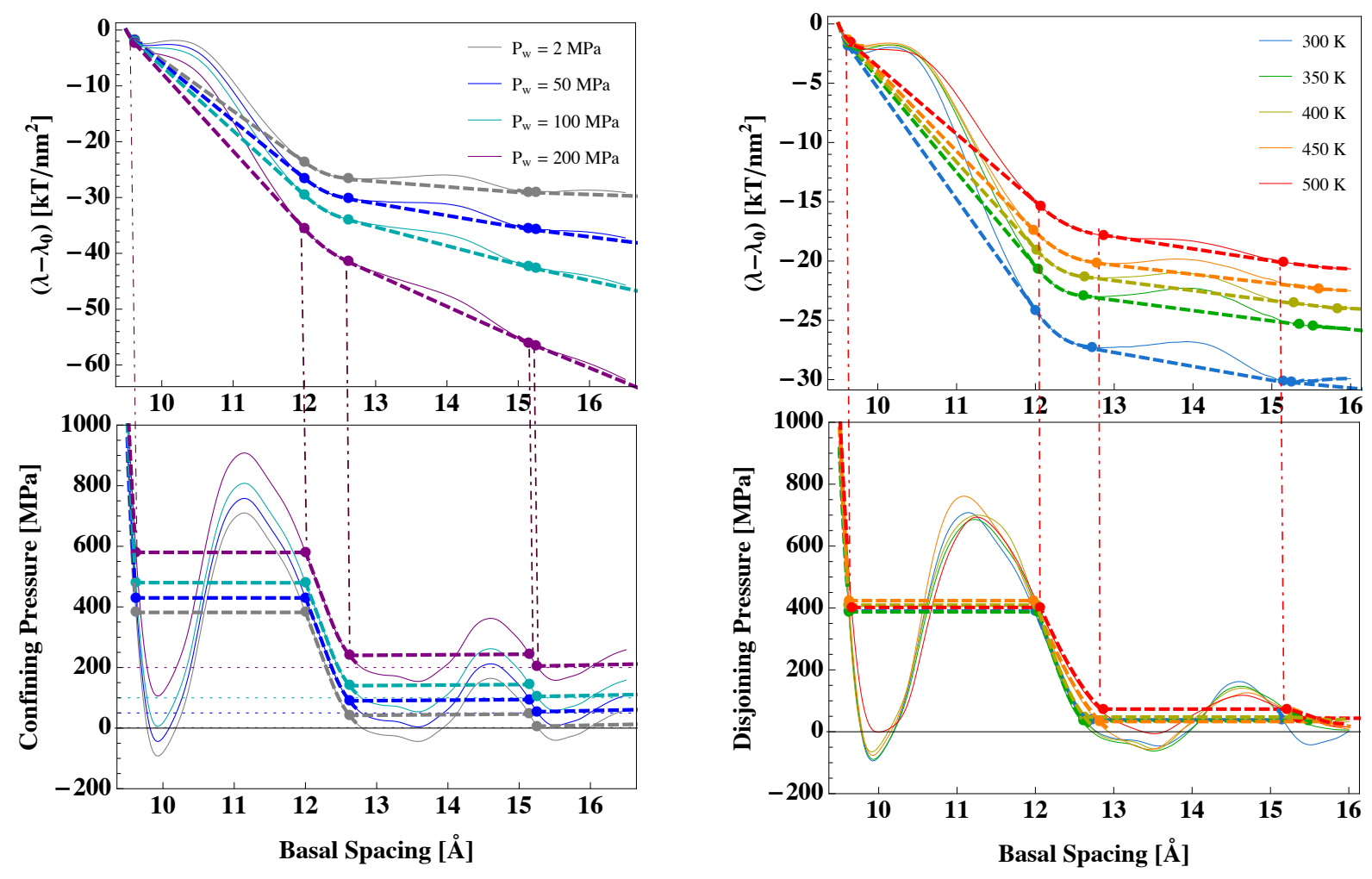

Figure 6: At left, free energy $\lambda$ at $300 \mathrm{~K}$ according to different water pressures $P_{w}$. The convex hull in energy profiles and the stable path in pressure isotherms are shown with dashed lines. At the bottom, the pressure isotherms and the stable paths are displayed. In the figure at the bottom, dotted lines represent the water pressure $P_{w}=10 \mathrm{MPa}$. At right, free energy $\lambda$ at different temperatures. The convex hull in energy profiles and the stable path in pressure isotherms are shown with dashed lines. At the bottom, the pressure isotherms and the stable paths are shown.

The free energy of swelling (ref. Eq. 4) of 0W/1W transition is shown in Figure 7 at both 300 and $500 \mathrm{~K}$ with water pressure $P_{w}=10 \mathrm{MPa}$ for pressures in the domain of metastability 
Table 1: Pressure at stable transitions, $P_{\text {Sta }}[\mathrm{MPa}]$

\begin{tabular}{|c|c|c|c|c|c|}
\hline & $300 \mathrm{~K}$ & $350 \mathrm{~K}$ & $400 \mathrm{~K}$ & $450 \mathrm{~K}$ & $500 \mathrm{~K}$ \\
\hline $0 \mathrm{~W} / 1 \mathrm{~W}\left(P_{w}=10 \mathrm{MPa}\right)$ & 389.9 & 386.9 & 408.9 & 423.7 & 399.6 \\
$0 \mathrm{~W} / 1 \mathrm{~W}\left(P_{w}=100 \mathrm{MPa}\right)$ & 479.9 & 477.1 & 498.9 & 513.5 & 488.9 \\
\hline $1 \mathrm{~W} / 2 \mathrm{~W}\left(P_{w}=10 \mathrm{MPa}\right)$ & 38.6 & 44.4 & 48.0 & 43.8 & 77.0 \\
$1 \mathrm{~W} / 2 \mathrm{~W}\left(P_{w}=100 \mathrm{MPa}\right)$ & 128.6 & 134.3 & 137.0 & 135.3 & 150.6 \\
\hline
\end{tabular}

$\left[P_{S p i}^{+}, P_{S p i}^{-}\right]$. Stable phase transition occurs for the pressure $P_{S t a}$ at which the two minima are equal; the other situations are metastable. The energy barriers at $0 \mathrm{~W} / 1 \mathrm{~W}$ and $1 \mathrm{~W} / 2 \mathrm{~W}$ transitions under a water pressure $P_{w}$ of $10 \mathrm{MPa}$ are depicted in Figure 8 according to the temperature and confining pressure. The energy barrier $\Delta \lambda^{*}$ reaches a maximum at $P_{S t a}$ and vanishes at $P_{S p i}^{+}$and $P_{S p i}^{-} ; \Delta \lambda^{*}$ decreases with the temperature and is larger for $0 \mathrm{~W} / 1 \mathrm{~W}$ than $1 \mathrm{~W} / 2 \mathrm{~W}$ state transitions. In agreement with Tambach et al., ${ }^{14}$ the energy barriers associated with hydration state transitions of montmorillonites have the order of magnitude of a few $k T / \mathrm{nm}^{2}$.

The maximum energy barrier for a state transition evolves almost linearly, within the range of temperature considered, as shown in the inset of Figure 8. A line can be fitted to each curve and the rate of variation of the maximum $\Delta \lambda^{*}$ with the temperature is -0.0152 and $-0.0068 \mathrm{kT} / \mathrm{nm}^{2} / \mathrm{K}$ for $0 \mathrm{~W} / 1 \mathrm{~W}\left(\mathrm{R}^{2}=0.99\right)$ and $1 \mathrm{~W} / 2 \mathrm{~W}\left(\mathrm{R}^{2}=0.94\right)$ transitions, respectively. The (metastable) energy barrier decreases more rapidly with a given increment of pressure favoring the $1 \mathrm{~W} \rightarrow 0 \mathrm{~W}$ transition with respect to the $0 \mathrm{~W} \rightarrow 1 \mathrm{~W}$ transition. In other words, for the same (absolute) increment of confining pressure $|\delta P|, \Delta \lambda^{*}$ is smaller in compression loading $(1 \mathrm{~W} \rightarrow 0 \mathrm{~W})$ than in unloading $(0 \mathrm{~W} \rightarrow 1 \mathrm{~W})$. This effect is less pronounced in $1 \mathrm{~W} / 2 \mathrm{~W}$ transitions. Applying transition state theory, for a given surface area of clay layer, $1 \mathrm{~W} \rightarrow 0 \mathrm{~W}$ transition is expected to occur faster than $0 \mathrm{~W} \rightarrow 1 \mathrm{~W}$ transition. This asymmetry decreases with the temperature. The ratio $\exp \left[-\Delta \Lambda_{(1 W \rightarrow 0 W)}^{*} /(k T)\right] / \exp \left[-\Delta \Lambda_{(0 W \rightarrow 1 W)}^{*} /(k T)\right]$ uncovers this asymmetry, as shown in the top-right inset of Figure 8. This ratio can be fitted with $\exp [A|\delta P|]$ with $A=0.093 \mathrm{MPa}^{-1}$ for $300 \mathrm{~K}, 0.074 \mathrm{MPa}^{-1}$ for $400 \mathrm{~K}$ and $0.058 \mathrm{MPa}^{-1}$ for $500 \mathrm{~K}$. 

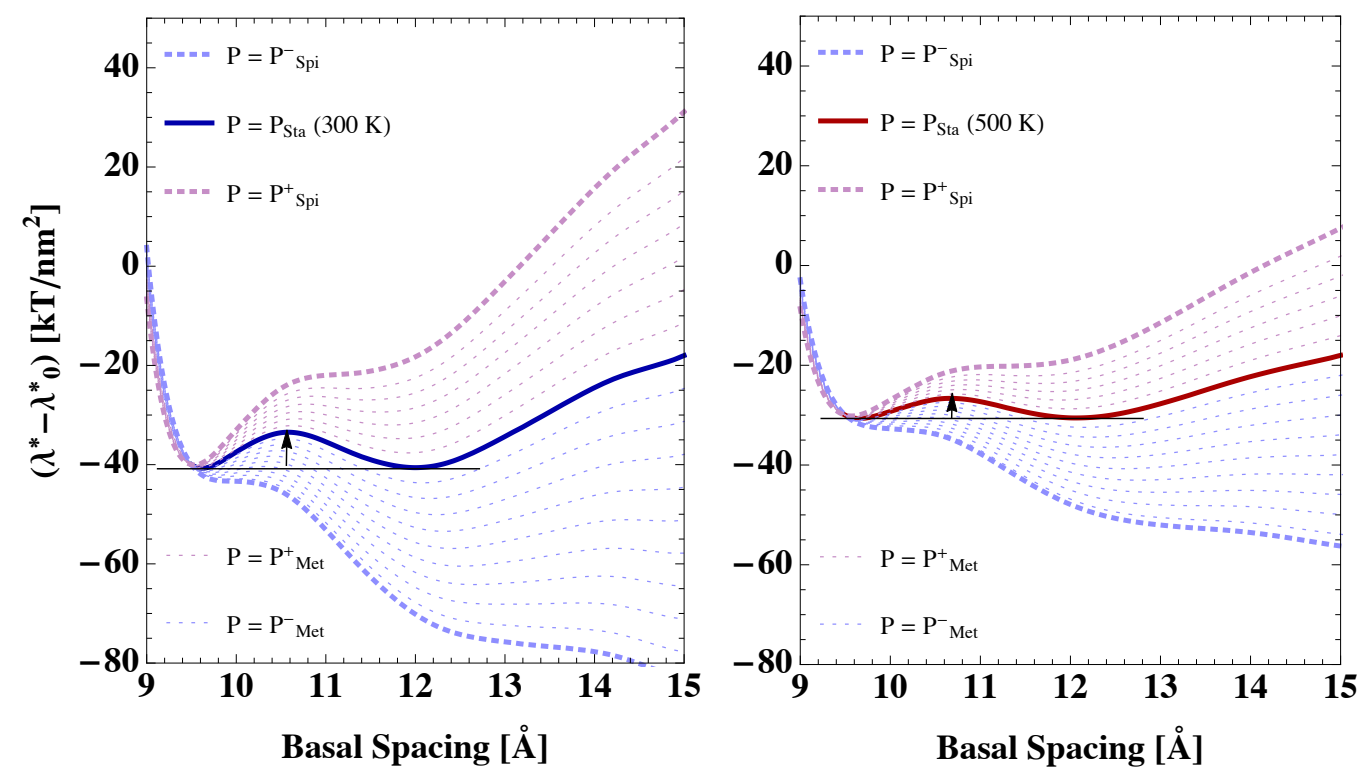

Figure 7: Free energy of swelling of $0 \mathrm{~W} / 1 \mathrm{~W}$ transition at $300 \mathrm{~K}$ (left) and $500 \mathrm{~K}$ (right) according to different confining pressures with water pressure $P_{w}=10 \mathrm{MPa}$. The solid lines are the free energy associated with the stable phase transition $\left(P_{S t a}\right)$ - the energy barrier to be overcome in the transition is depicted in the graphic. The dashed curves $P_{S p i}^{+}$and $P_{S p i}^{-}$correspond to the pressures at spinodal decomposition for compressive loading and unloading, respectively. The thin dashed curves refer to intermediate pressures $P_{M e t}$, in metastable domain, in increments of $50 \mathrm{MPa}$ with respect to $P_{S t a} .|\delta P|$ is the increment of confining pressure. 


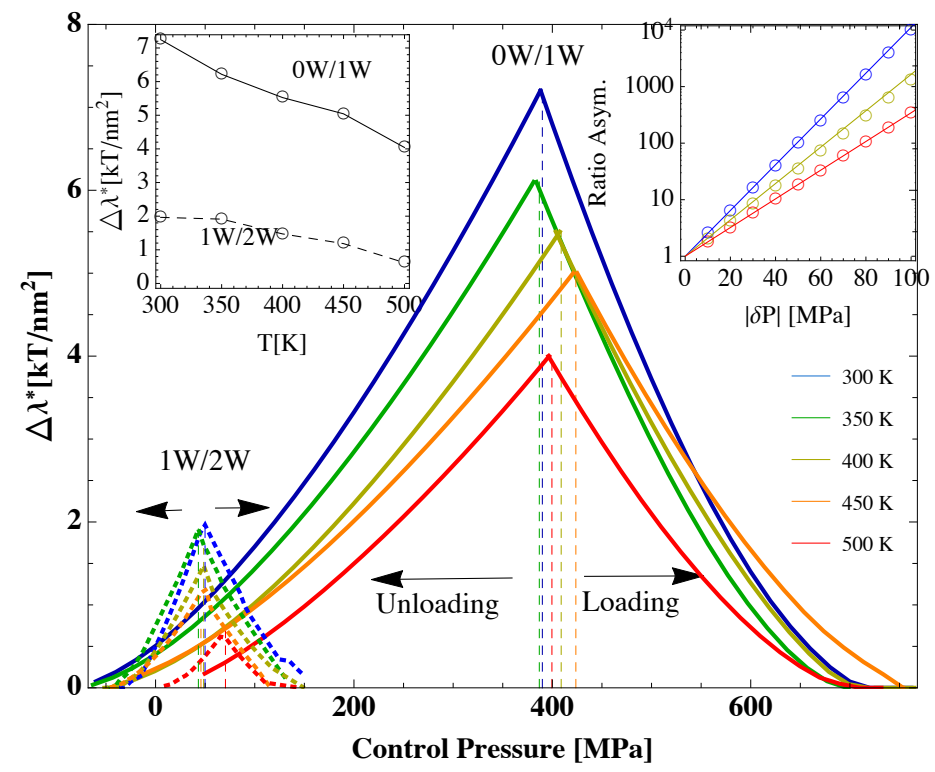

Figure 8: Energy barriers $\Delta \lambda^{*}$ at $0 \mathrm{~W} / 1 \mathrm{~W}$ and $1 \mathrm{~W} / 2 \mathrm{~W}$ transitions at water pressure $P_{w}=$ $10 \mathrm{MPa}$ according to the temperature and pressure (in a system controlled by the confining pressure). The left inset displays the evolution of the maximum $\Delta \lambda^{*}$ (associated with $P_{S t a}$ ) with the temperature. The right inset shows the kinetic asymmetry under loading and unloading expressed in terms of $\exp \left[-\Delta \Lambda_{(1 W \rightarrow 0 W)}^{*} /(k T)\right] / \exp \left[-\Delta \Lambda_{(0 W \rightarrow 1 W)}^{*} /(k T)\right]$ ratio as a function of the absolute increment of pressure $|\delta P|$; the circles are results from simulations and the solid lines are fittings with $\exp [A|\delta P|]$, with $A=0.092,0.068$ or $0.056 \mathrm{MPa}^{-1}$ for 300,400 and $500 \mathrm{~K}$, respectively. 


\section{Hydration phase diagrams}

The results discussed in the last section can be used to build a hydration phase diagram of NaMmt at the clay particle scale. The variables of interest are the confining pressure, pressure of fluid reservoir and the temperature. Figure 9 shows the isothermal hydration phase diagram of a Na-Mmt particle as a function of confining pressure $P$ and water pressure $P_{w}$ for different temperatures in a compressive loading. The phase boundaries move towards higher pressures with temperature increase in a way that the domains of metastability shrink. The confining pressure at which water is completely squeezed out from interlayer pores (boundary of $0 \mathrm{~W}$ state) is above $700 \mathrm{MPa}$, for a water pressure of $P_{w}=0 \mathrm{MPa}$ at $300 \mathrm{~K}$, if metastability is accounted for (so the limit of dehydration is the right-most dashed line instead of the solid line in the diagrams of Figure 9), which is appropriate for short timescale considerations. In geological timescales, the stable path is respected and the limit of dehydration decreases to $400 \mathrm{MPa}$ at $P_{w}=0 \mathrm{MPa}$ and $300 \mathrm{~K}$, which is a confining pressure that is encountered at a

depth of few kilometers in Earth's crust. ${ }^{10}$ Moreover, due to the heterogeneity of clay-rich rock and soils, fluctuations of stresses are expected within clays. In this way, the depth at which confining pressures are sufficiently large to dehydrate the clay might be shallower. It is noteworthy that the transitions $1 \mathrm{~W} / 2 \mathrm{~W}$ are more common for most of industrial and environmental processes involving clays since they occur at much lower pressures.

The phase diagram presented here does not include a $3 \mathrm{~W}$ swelling state since this state could not be clearly identified according to our results. This state would be located inbetween pore water $\infty \mathrm{W}$ and $2 \mathrm{~W}$ states, i.e. accompanying low confining pressures. In our simulations, the oscillations corresponding to $3 \mathrm{~W}$ state have the same order of magnitude of the fluctuations observed in the results. Note however that this state is routinely observed experimentally ${ }^{56}$ In the systems studied here, more precise simulations would be necessary to identify this hydration state. Other clay compositions, with different layer charge for instance, may also be more prone to yield $3 \mathrm{~W}$ state.

The same diagrams could be drawn for unloading, but except for the $0 \mathrm{~W} \rightarrow 1 \mathrm{~W}$ transition, 
the transition pressures are very close to the water pressure $P_{w}$ and the spinodal pressures could not be computed in a precise way. For some $P_{w}$, the spinodal pressures in unloading can be negative. The clay particles are expected to become unstable when $P<P_{w}$. Thus, we restrict our diagrams to compressive loadings.
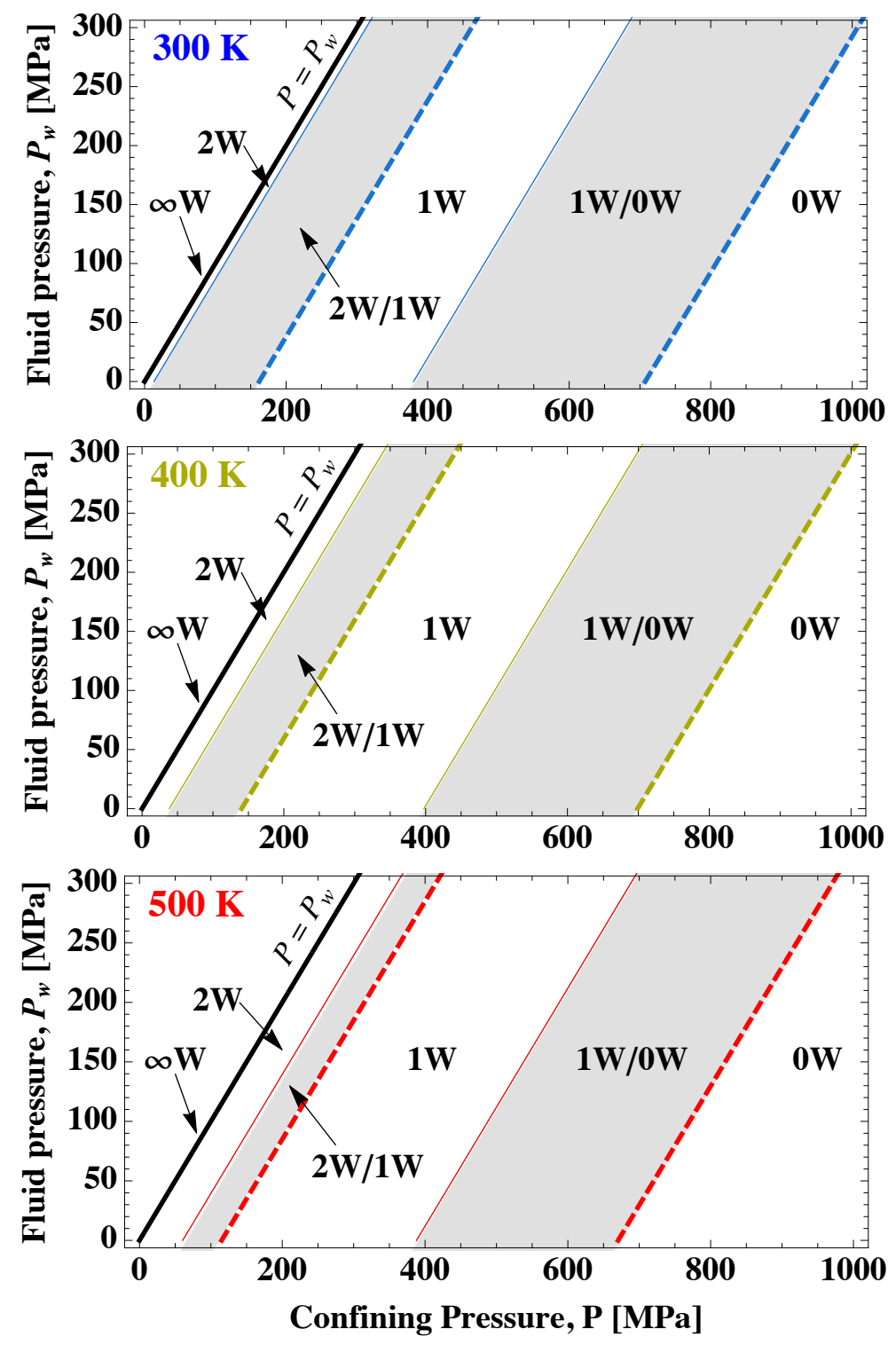

Figure 9: Hydration-phase diagram of a clay particle as a function of the confining pressure $P$ and fluid reservoir pressure $P_{w}$ for different temperatures in a compressive loading.

The hydration phase diagram for a fixed water pressure $P_{w}$ and varying temperature is 
shown in Figure 10. The confining pressure at which the particle completely dehydrates slightly decreases if metastability (i.e. $1 W / 0 W \rightarrow 0 W$, associated with short time scales) is accounted for (top-most dashed line). Similar behavior is observed for the transition $2 W / 1 W \rightarrow 1 W$, where metastability is also accounted for. In the absence of metastability (i.e. a scenario associated with long time scales), the confining pressure at which the particle completely dehydrates $(1 W \rightarrow 0 W$, top-most solid line) slightly increases with the temperature; the same occurs in stable $2 W \rightarrow 1 W$ transition. The domain of metastability decreases, therefore, with the temperature. In this diagram, we ignored geochemistry and possible conversion of montmorillonite in other clay minerals. Vidal and Dubacq, ${ }^{17}$ for example, provides phase diagrams for such conversions according to the temperature and compositional environments.

Since the mechanical and thermal properties of a clay particle are often a function of the hydration state of a stack, the hydration phase diagrams proposed in this section can be used as an input to multiscale analysis of the thermo-mechanical behavior of clays.

\section{Summary and Conclusions}

In this paper, we consider the hydration states of clays as phases and the transition between these states, as phase changes. The thermodynamic formulation supporting this idea is presented and results from molecular simulation of Na-Mmt are used in an application. The stability analysis presented here explains the coexistence of different hydration states at clay particle scale and provides elements to understand the irreversibilities of clay behavior associated with adsorption. Hysteresis is observed in the behavior of other swelling clays with different types of ion charge. ${ }^{14}$ It is noteworthy that once the swelling free energy profiles show a multi-well configuration, a certain degree of hysteresis is expected. Molecular simulations of different clays (e.g. ${ }^{14,35,55}$ ) employing different interatomic potentials, yields swelling free energy profiles with this multi-well character. As discussed in this paper, 


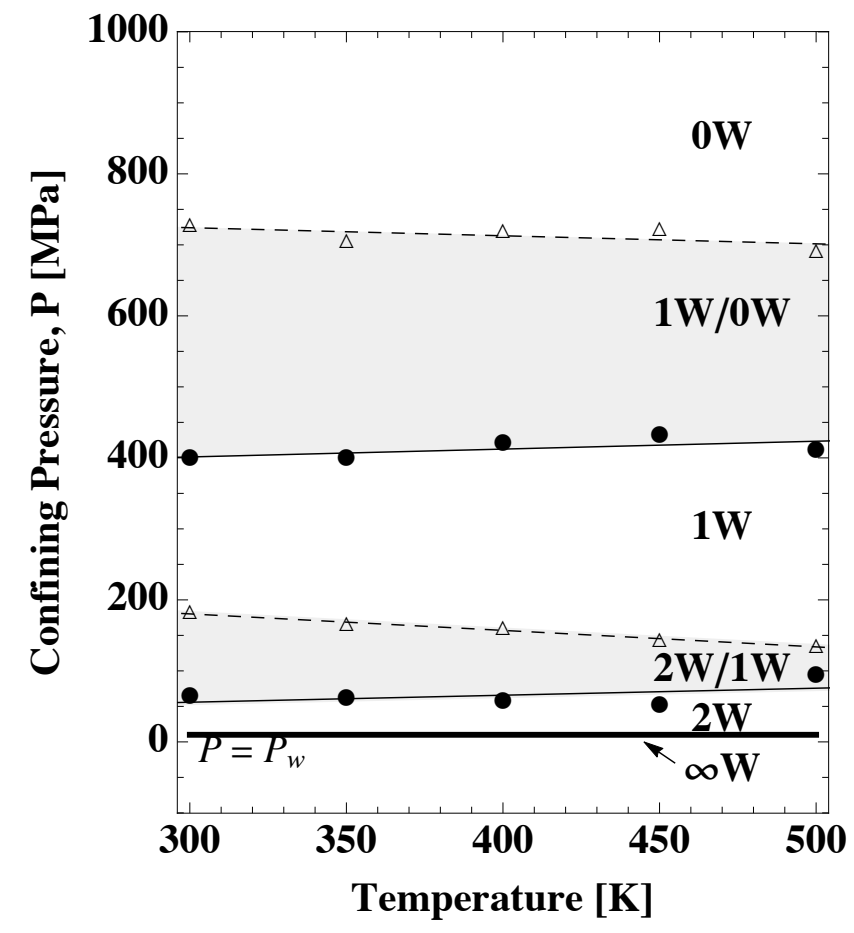

Figure 10: Hydration-phase diagram as a function of the confining pressure $P$ and temperature for water pressure $P_{w}=10 \mathrm{MPa}$. The lines following the points obtained from simulations (full circles for stable transitions and triangles for spinodal decomposition) are least-square linear. Note that above $373 \mathrm{~K}$, conversion to illite may occur. ${ }^{10}$ 
the prevalence of the states associated with the different energy wells will depend on the energy barriers separating the states. Such information provides interesting insights into the mechanical behavior of the elementary constituents of clays, which may be of interest for a better understanding of the macroscopic behavior of clay-rich rocks and soils. In this sense, we provide a contribution to fill the gap between the aspects revealed by investigation at the molecular scale to the "continuum" mechanics of clays, as used in geomechanics.

We conclude:

- In saturated conditions (i.e. pressure of water higher than the pressure of liquid saturation of water at a given temperature), the disjoining pressure isotherms for Na-Mmt are negligibly affected by variations of the pressure of water reservoir. Thus, confining pressure isotherms under different pressures of water reservoirs can be obtained from a single pressure isotherm. This aspect translates in the range of basal spacings associated with (meta-)stability being weakly affected by the pressure of water reservoir.

- The temperature slightly reduces the range of confining pressures associated with metastability within a clay particle, as observed previously in a basic model of layered adsorbing materials. ${ }^{6}$ The energy barriers to be overcome in phase transitions decrease linearly with the temperature at a rate on the order of magnitude of 0.01 $k T / \mathrm{nm}^{2} / \mathrm{K}$ for both $0 \mathrm{~W} / 1 \mathrm{~W}$ and $1 \mathrm{~W} / 2 \mathrm{~W}$ transitions. A kinetic asymmetric behavior is noticed with respect to compressive loading and unloading. This asymmetry is more pronounced for $0 \mathrm{~W} / 1 \mathrm{~W}$ transitions and decreases with the temperature.

- Hydration phase diagrams could be built based on the analysis performed in this paper. We provide hydration phase diagrams updating previous diagrams, ${ }^{10}$ which were based only on continuum thermodynamics. For a given water pressure, the confining pressure in hydration phase diagrams associated with a transition of hydration states decreases with the temperature. Our phase diagrams describe saturated clays in a range of confining pressures, pressures of water reservoir and temperatures which are relevant 
for geomechanical applications. These diagrams can be used as an input to multiscale analysis of the thermo-mechanical behavior of clays so that a link between adsorption phenomena (associated with the molecular scale) and the macroscopic behavior of clays can be established.

\section{Acknowledgement}

The financial support of the French National Research Agency (ANR) through the project TEAM2ClayDesicc (ANR-14-CE05-0023-01) is gratefully acknowledged.

\section{References}

(1) Meunier, A. Clays; Springer Berlin Heidelberg, 2005; pp 295-327, DOI: 10.1007/3-54027141-4_7.

(2) Ferrage, E.; Lanson, B.; Malikova, N.; PlanÃğon, A.; Sakharov, B. A.; Drits, V. A. New Insights on the Distribution of Interlayer Water in Bi-Hydrated Smectite from X-ray Diffraction Profile Modeling of 001 Reflections. Chemistry of Materials 2005, 17, 3499-3512.

(3) Ferrage, E. Investigation of the Interlayer Organization of Water and Ions In Smectite from the Combined Use of Diffraction Experiments And Molecular Simulations. a Review of Methodology, Applications, And Perspectives. Clays and Clay Minerals 2016, $64,348-373$.

(4) Ebrahimi, D.; Whittle, A. J.; Pellenq, R. J.-M. Effect of Polydispersity of Clay Platelets on the Aggregation And Mechanical Properties of Clay at the Mesoscale. Clays and Clay Minerals 2016, 64, 425-437. 
(5) Churakov, S. V.; Gimmi, T. Up-Scaling of Molecular Diffusion Coefficients in Clays: A Two-Step Approach. The Journal of Physical Chemistry C 2011, 115, 6703-6714.

(6) Brochard, L.; Hon Âşrio, T.; Vandamme, M.; Bornert, M.; Peigney, M. Nanoscale origin of the thermo-mechanical behavior of clays. Acta Geotechnica 2017, in press, 1-19.

(7) Brochard, L.; Honorio, T.; Vandamme, M.; Stefanou, I.; Ghabezloo, S.; Bornert, M. A Possible Nano-Scale Origin of the Surprising Thermal Expansion of Clays. Poromechanics VI 2017, 626-633.

(8) Verburg, K.; Baveye, P.; McBride, M. B. Cation-exchange hysteresis and dynamics of formation and breakdown of montmorillonite quasi-crystals. Soil Science Society of America Journal 1995, 59, 1268-1273.

(9) Boek, E. S.; Coveney, P. V.; Skipper, N. T. Molecular Modeling of Clay Hydration: A Study of Hysteresis Loops in the Swelling Curves of Sodium Montmorillonites. Langmuir 1995, 11, 4629-4631.

(10) Bird, P. Hydration-phase diagrams and friction of montmorillonite under laboratory and geologic conditions, with implications for shale compaction, slope stability, and strength of fault gouge. Tectonophysics 1984, 10\%, 235-260.

(11) Laird, D. A.; Shang, C. Relationship between cation exchange selectivity and crystalline swelling in expanding 2: 1 phyllosilicates. Clays and Clay Minerals 1997, 45, 681-689.

(12) Wada, N.; Hines, D. R.; Ahrenkiel, S. P. X-ray-diffraction studies of hydration transitions in Na vermiculite. Physical Review B 1990, 41, 12895-12901.

(13) da Silva, G. J.; Fossum, J. O.; DiMasi, E.; MÃělÃ̈̈y, K. J. Hydration transitions in a nanolayered synthetic silicate: A synchrotron x-ray scattering study. Physical Review B 2003, 67, 094114. 
(14) Tambach, T. J.; Bolhuis, P. G.; Hensen, E. J. M.; Smit, B. Hysteresis in Clay Swelling Induced by Hydrogen Bonding:âĂL Accurate Prediction of Swelling States. Langmuir 2006, 22, 1223-1234.

(15) Ferrage, E.; Lanson, B.; Michot, L. J.; Robert, J.-L. Hydration Properties and Interlayer Organization of Water and Ions in Synthetic Na-Smectite with Tetrahedral Layer Charge. Part 1. Results from X-ray Diffraction Profile Modeling. The Journal of Physical Chemistry C 2010, 114, 4515-4526.

(16) Emmerich, K.; Koeniger, F.; Kaden, H.; Thissen, P. Microscopic structure and properties of discrete water layer in Na-exchanged montmorillonite. Journal of Colloid and Interface Science 2015, 448, 24-31.

(17) Vidal, O.; Dubacq, B. Thermodynamic modelling of clay dehydration, stability and compositional evolution with temperature, pressure and $\mathrm{H} 2 \mathrm{O}$ activity. Geochimica et Cosmochimica Acta 2009, 73, 6544-6564.

(18) Boek, E. S.; Coveney, P. V.; Skipper, N. T. Molecular Modeling of Clay Hydration: A Study of Hysteresis Loops in the Swelling Curves of Sodium Montmorillonites. Langmuir 1995, 11, 4629-4631.

(19) Ngouana W., B. F.; Kalinichev, A. G. Structural Arrangements of Isomorphic Substitutions in Smectites: Molecular Simulation of the Swelling Properties, Interlayer Structure, and Dynamics of Hydrated CsâĂŞMontmorillonite Revisited with New Clay Models. The Journal of Physical Chemistry C 2014, 118, 12758-12773.

(20) Holmboe, M.; Bourg, I. C. Molecular Dynamics Simulations of Water and Sodium Diffusion in Smectite Interlayer Nanopores as a Function of Pore Size and Temperature. The Journal of Physical Chemistry C 2014, 118, 1001-1013.

(21) Teich-McGoldrick, S. L.; Greathouse, J. A.; JovÃl-ColÃşn, C. F.; Cygan, R. T. Swelling Properties of Montmorillonite and Beidellite Clay Minerals from Molecular Simulation: 
Comparison of Temperature, Interlayer Cation, and Charge Location Effects. The Journal of Physical Chemistry C 2015, 119, 20880-20891.

(22) de Lourdes ChÃąvez, M.; de Pablo, L.; de Pablo, J. J. Monte Carlo Molecular Simulation of the Hydration of K-Montmorillonite at $353 \mathrm{~K}$ and 625 bar. Langmuir 2004, 20, 10764-10770.

(23) Shroll, R. M.; Smith, D. E. Molecular dynamics simulations in the grand canonical ensemble: Application to clay mineral swelling. The Journal of Chemical Physics 1999, 111, 9025-9033.

(24) Underwood, B. S.; Kim, Y. R. Effect of volumetric factors on the mechanical behavior of asphalt fine aggregate matrix and the relationship to asphalt mixture properties. Construction and Building Materials 2013, 49, 672-681.

(25) Carrier, B. Influence of water on the short-term and long-term mechanical properties of swelling clays: experiments on self-supporting films and molecular simulations. $\mathrm{PhD}$ Thesis, Universit Ãl' Paris-Est, 2013.

(26) Carrier, B.; Vandamme, M.; Pellenq, R. J.-M.; Van Damme, H. Elastic Properties of Swelling Clay Particles at Finite Temperature upon Hydration. The Journal of Physical Chemistry C 2014, 118, 8933-8943.

(27) Lee, J. H.; Guggenheim, S. Single crystal X-ray refinement of pyrophyllite-1Tc. American Mineralogist 1981, 66, 350-357.

(28) Loewenstein, W. The distribution of aluminum in the tetrahedra of silicates and aluminates. American Mineralogist 1954, 39, 92-96.

(29) Cygan, R. T.; Liang, J.-J.; Kalinichev, A. G. Molecular Models of Hydroxide, Oxyhydroxide, and Clay Phases and the Development of a General Force Field. The Journal of Physical Chemistry B 2004, 108, 1255-1266. 
(30) Berendsen, H. J. C.; Grigera, J. R.; Straatsma, T. P. The missing term in effective pair potentials. The Journal of Physical Chemistry 1987, 91, 6269-6271.

(31) Plimpton, S. Fast Parallel Algorithms for Short-Range Molecular Dynamics. Journal of Computational Physics 1995, 117, 1-19.

(32) Martin, M. G. MCCCS Towhee: a tool for Monte Carlo molecular simulation. Molecular Simulation 2013, 39, 1212-1222.

(33) Allen, M. P.; Tildesley, D. J. Computer Simulation of Liquids; Oxford University Press: New York, 1989.

(34) Suter, J. L.; Coveney, P. V.; Greenwell, H. C.; Thyveetil, M.-A. Large-Scale Molecular Dynamics Study of Montmorillonite Clay:âĂL' Emergence of Undulatory Fluctuations and Determination of Material Properties. The Journal of Physical Chemistry C 2007, $111,8248-8259$.

(35) Smith, D. E.; Wang, Y.; Chaturvedi, A.; Whitley, H. D. Molecular Simulations of the Pressure, Temperature, and Chemical Potential Dependencies of Clay Swelling. The Journal of Physical Chemistry B 2006, 110, 20046-20054.

(36) NIST, SAT-TMMC: Liquid-Vapor coexistence properties - SPC/E Water. 2014; http: //www.nist.gov/mml/csd/informatics_research/spce_water_sat_lrc.cfm\#refs, updated November 22, 2016.

(37) Botan, A.; Rotenberg, B.; Marry, V.; Turq, P.; Noetinger, B. Hydrodynamics in Clay Nanopores. The Journal of Physical Chemistry C 2011, 115, 16109-16115.

(38) Whitley, H. D.; Smith, D. E. Free energy, energy, and entropy of swelling in Cs-, Na-, and Sr-montmorillonite clays. The Journal of Chemical Physics 2004, 120, 5387-5395.

(39) Bousquet, D.; Coudert, F.-X.; Fossati, A. G. J.; Neimark, A. V.; Fuchs, A. H.; Boutin, A. Adsorption induced transitions in soft porous crystals: An osmotic poten- 
tial approach to multistability and intermediate structures. The Journal of Chemical Physics 2013, 138, 174706.

(40) Bhattacharya, K. Theory of martensitic microstructure and the shape-memory effect; 2003.

(41) James, R. D. Displacive phase transformations in solids. Journal of the Mechanics and Physics of Solids 1986, 34, 359-394.

(42) Salles, F.; Bildstein, O.; Douillard, J. M.; Jullien, M.; Raynal, J.; Van Damme, H. On the Cation Dependence of Interlamellar and Interparticular Water and Swelling in Smectite Clays. Langmuir 2010, 26, 5028-5037.

(43) Drits, V.; Eberl, D.; Srodon, J. XRD measurement of mean thickness, thickness distribution and strain for illite and illite-smectite crystallites by the Bertaut-WarrenAverbach technique. Clays and Clay Minerals 1998, 46, 38-50.

(44) Mystkowski, K.; Srodon, J.; Elsass, F. Mean thickness and thickness distribution of smectite crystallites. Clay Minerals 2000, 35, 545-557.

(45) Jonas, E. C.; Oliver, R. M. Size and shape of montmorillonite crystallites. Clays Clay Miner 1967, 15, 27-33.

(46) Beermann, T.; Brockamp, O. Structure analysis of montmorillonite crystallites by convergent-beam electron diffraction. Clay Minerals 2016, 40, 1-13.

(47) Nadeau, P. H. The physical dimensions of fundamental clay particles. CLAY MINER. Clay Miner. 1985, 20, 499.

(48) Ballard, D. G. H.; Rideal, G. R. Flexible inorganic films and coatings. Journal of Materials Science 1983, 18, 545-561.

(49) Honorio, T.; Brochard, L. Flexibility of C-S-H sheets and stacks from molecular simulations. 2017. 
(50) Honorio, T.; Brochard, L.; Vandamme, M. Flexibility of clay layers and implications for the nanotexture. (submitted paper) 2017,

(51) Honorio, T.; Brochard, L.; Vandamme, M.; Stefanou, I.; Ghabezloo, S.; Bornert, M. Stability of Hydrated Clay Layers from Molecular Simulations. Poromechanics VI 2017, $688-695$.

(52) Tamura, K.; Setsuda, H.; Taniguchi, M.; Yamagishi, A. Application of the LangmuirâĹŠBlodgett Technique to Prepare a ClayấĽSMetal Complex Hybrid Film. Langmuir 1999, 15, 6915-6920.

(53) Truhlar, D. G.; Isaacson, A. D.; Garrett, B. C. Generalized transition state theory. Theory of chemical reaction dynamics 1985, 4, 65-137.

(54) Skipper, N. T.; Chang, F.-R. C.; Sposito, G. Monte Carlo simulation of interlayer molecular structure in swelling clay minerals. I: Methodology. Clays and clay minerals 1995, 43, 285-293.

(55) Karaborni, S.; Smit, B.; Heidug, W.; Urai, J. The Swelling of Clays: Molecular Simulations of the Hydration of Montmorillonite. Science 1996, 271, 1102-1104.

(56) Holmboe, M.; Wold, S.; Jonsson, M. Porosity investigation of compacted bentonite using XRD profile modeling. Journal of contaminant hydrology 2012, 128, 19-32.

(57) Coussy, O. Mechanics and Physics of Porous Solids; Wiley, 2010.

(58) Brochard, L.; Vandamme, M.; Pellenq, R. J. M. Poromechanics of microporous media. Journal of the Mechanics and Physics of Solids 2012, 60, 606-622.

(59) Cariou, S. Couplage hydro-mÃl'canique et transfert dans l'argilite de Meuse/HauteMarne : approches exp Âl'rimentale et multi-Âl'chelle. phdthesis, Ecole des Ponts ParisTech, 2010 . 
(60) Roth, A. Savitzky-Golay Smoothing Filters. 2000; http://library.wolfram.com/ infocenter/MathSource/789/. 


\section{Graphical TOC Entry}

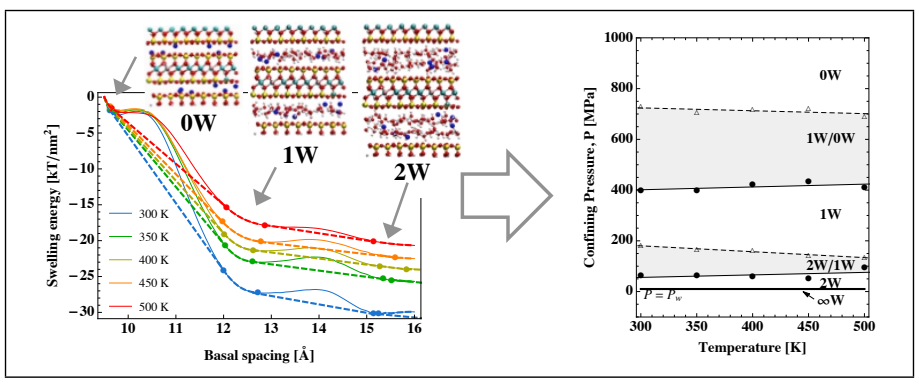

